\title{
Dünnschichtchromatographische Trennung von Ketocarbonsäuren im Harn von Patienten mit Phenylketonurie und Cystinose
}

\author{
Von P. Lutz und G. M. v. REUtern
}

Aus der Kinderklinik der Universität Heidelberg (Direktor: Prof. Dr. H. Bickel)

(Eingegangen am 11. Juni 1969)

Es wird ein dünnschichtchromatographisches Verfahren zur Trennung der 2,4-Dinitro-phenylhydrazone von Ketocarbonsäuren beschrieben. Mit dieser Methode wurden Urine normaler Personen sowie Patienten mit Phenylketonurie und Cystinose untersucht. Bei beiden Krankheiten wurden typische Ausscheidungsmuster gefunden; im Falle der Phenylketonurie bildete sich dieses unter erfolgreicher Behandlung zurück. Es wird ein Suchtest angegeben.

\section{Thin layer cbromatographic separation of keto acids in the urine of patients with phenylketonuria and cystinosis}

A method is described for the separation of the keto acids by thin layer chromatography after converting them into the corresponding 2,4-dinitro-phenylhydrazones. We have analyzed the keto acids in the urine of normal persons and of patients with phenylketonuria and cystinosis and have found in these diseases a typical chromatographic pattern. This picture disappears in phenylketonuria under treatment. A screening test is described.

Ketocarbonsäuren, im folgenden einfach Ketosäuren genannt, treten an verschiedenen Stellen des intermediären Stoffwechsels auf, so im Citronensäurezyklus, bei der oxydativen Desaminierung von Aminosäuren und deren weiterem Abbau. Serumkonzentration und Ausscheidung der entsprechenden Ketosäuren können daher bei Störungen des Aminosäurestoffwechsels, wie z. B. der Phenylketonurie, verändert sein. Bei der Cystinose sind aus ungeklärten Gründen im Serum und Urin Ketosäuren vermehrt, die mit dem Citronensäurezyklus in Zusammenhang stehen. Durch die Bestimmung des Ketosäuremusters der Körperflüssigkeiten können daher Krankheiten erkannt und eventuell Stoffwechselstörungen unbekannter Genese geklärt werden. Die bisher üblichen Verfahren zur Bestimmung von Ketosäuren lassen sich wie folgt einteilen:

1. Bildung langwellig absorbierender Komplexe mit Eisen und Bor

2. Fällungen (Hydrazone)

3. Enzymatische Reaktionen

4. Chromatographische Trennung von Derivaten

Die Reaktionen unter 1. und 2. erfassen nur die Gesamtheit der Ketosäuren, die enzymatischen Methoden erlauben nur die Bestimmung jeweils einer Ketosäure. 1949 beschrieben Cavalirns, Frontali und Toschi (1) erstmals eine Auftrennung von Ketosäuren mit eindimensionaler Papierchromatographie nach deren Umwandlung in 2,4-Dinitro-phenylhydrazone. Die leicht zersetzlichen Ketosäuren sind dabei in die gelb gefärbten und stabilen Hydrazone umgewandelt, die sich auf dem Chromatogramm ohne weitere Anfärbung erkennen lassen. Durch cis-trans-Isomerie entstehende Doppelflecken erschweren die Identifizierung. Die Extraktion der Ketosäuren ist langwierig. Zahlreiche Versuche wurden daher unternommen, die ursprüngliche Methode von Cavallini, Frontali und Toschi zu vereinfachen und weitere Ketosäuren gegenüber der von ihnen gefundenen 2-Oxo-glutarsãure und Brenztraubensäure abzutrennen. So gelang es z. B. Coward und SMITH (2) mit zweidimensionaler Papierchromatographie, zahlreiche Flecke bei der Untersuchung von Urin, Plasma, Speichel und Schweiß voneinander zu trennen, ohne sie im einzelnen zu identifizieren können. BERLET (3) trennte kürzlich die Hydrazone von sechs Ketosäuren zweidimensional auf Kieselgel. Untersuchungen größerer Gruppen gesunder und kranker Personen liegen unseres Wissens noch nicht vor. Zweidimensionale Chromatographie auf Dünnschichtplatten erschien uns am geeignetsten, da es nicht möglich ist, eindimensional alle Flecken zu trennen, und da die Dünnschichtchromatographie wesentlich einfacher zu handhaben ist als die Papierchromatographie.

\section{Material und Methoden}

Vergleichssubstanzen sind zur Identifizierung unbekannter Flecken und Testung von Fließmitteln und Sorptionsschichten nötig. Im Handel erwarben wir: Glyoxylsäure, Brenztraubensäure, Oxalessigsäure, 2-Oxo-glutarsäure, Oxalbernsteinsäure, 2-Oxo-buttersäure, Ascorbinsäure, Laevulinsäure (4Oxo-n-valeriansäure), Phenylbrenztraubensäure, 4-Hydroxy-phenylbrenztraubensäure, 4-Methyl-2-oxo-pentansäure, 2-Oxo-n-valeriansäure.

3-Methyl-2-oxo-pentansäure und 2-Oxo-4-methylmercaptobuttersäure stellten wir enzymatisch nach MEISTER (4) aus den entsprechenden Aminosäuren dar. Die Elementaranalyse ergab mit den errechneten übereinstimmende Werte. Es gelang uns nicht, auf diese Weise die 2-Oxo-3-mercapto-propionsäure aus Cystein herzustellen. Mit den Ketosäuten stellten wir nach HAWARY und Thomson (5) die Hydrazone dar. Oxalbernsteinsäure wurde bei diesem Vorgang zu 2-Oxo-glutarsäure decarboxyliert, wie die Elementaranalyse und die $R_{\mathrm{F}}$-Werte zeigten. Die Schmelzpunkte stimmten mit den bei MEISTER (6) angegebenen überein.

\section{Fließmittel}

Mit den in Ăthylacetat gelösten Hydrazonen testeten wir eine Reihe von Fließmitteln und deren Kombinationen sowie Fertig- 
platten mit Cellulosebeschichtung und Kieselgelbeschichtung verschiedener Fabrikation. Die besten Ergebnisse erzielten wir auf Kieselgel-Fertigplatten der Firma Merck $(10 \times 10 \mathrm{~cm})$ (Charge Nr. 8488128) mit folgender Fließmittelkombination:

\section{Laufrichtung:}

Aceton/Isoamylalkohol/Wasser; 50:50:1 v/v

2. Laufrichtung:

Isoamylalkohol/0,25 $\mathrm{NH}_{4} \mathrm{OH} ; 20: 1 \mathrm{v} / \mathrm{v}$

Chloroform/Eisessig; 100:7 v/v (zwei Läufe).

Nach jedem der vier Läufe wird gut abgetrocknet. Wir trugen links unten jeweils $1 \mathrm{~cm}$ vom Plattenrand entfernt mit Mikrocaps auf, ließen die Platten etwa $0,5 \mathrm{~cm}$ eintauchen und die Fließmittel jeweils $9,5 \mathrm{~cm}$ hoch steigen.

\section{Fällung und Extraktion}

Die Fällung mit 0,2proz. 2,4-Dinitrophenylhydrazin in 2N Salzsäure aus dem Harn exfaßt Carbonylverbindungen ohne und mit Carboxylgruppe; letztere müssen in der folgenden Extraktion abgetrennt werden. Wir gingen im einzelnen so vor: $50 \mathrm{~m} / \mathrm{Harn}$ werden mit $25 \mathrm{ml}$ Reagenz versetzt, dann mit insgesamt $70 \mathrm{ml}$ Athylacetat in 4 Teilen im Scheidetrichter ausgeschüttelt. Die vereinigten organischen Phasen extrahierten wir mit $65 \mathrm{ml} 0,2$ proz. wäßr. $\mathrm{NaHCO}_{3}$-Lösung in 9 Teilen, wodurch die sauren Komponenten in die wäßr. Phase überführt werden. Nach Ansäuern mit $1 \mathrm{~N}$ Salzsäure werden diese wieder in $40 \mathrm{~m} l$ Äthylacetat in 4 Teilen aufgenommen. Die vereinigten organischen Phasen werden bis zur Trockene im Rotationsverdampfer eingedampft. Bei Emulsionsbildung zentrifugierten wir. Die Zentrifugationszeit konnte verkürzt werden, wenn wir zu Beginn der Aufarbeitung $2 \mathrm{~m} / \mathrm{n}$-Butanol zugaben.

\section{Bezugsgrößse}

Für die quantitative Auswertung bestimmten wir die Kreatininkonzentration jeder Probe nach JAfFé und trugen dann Volumina entsprechend 0,1 bis $0,5 \mathrm{mg}$ Kreatinin auf die Platten auf. Dazu lösten wir den bis zur Trockene eingedampften Extrakt in 0,5 bis $2 \mathrm{ml}$ Äthylacetat (z. B.: Löst man bei einem Harn-Kreatinin von $100 \mathrm{mg} / 100 \mathrm{ml}$ den Rückstand in $0,5 \mathrm{ml}$ Äthylacetat, so entspricht $1 \mu /$ Probenlösung 0,1 mg Kreatinin). Dann bleiben die Auftragsvolumina unter $20 \mu \mathrm{l}$. Der Bezug auf die Kreatininausscheidung erfolgte in Analogie zur dünnschichtchromatographischen Bestimmung der Aminosäuren (7).

\section{Sucbtest}

In einer abgekürzten Methode untersuchten wir die Harne von 25 gesunden und kranken Kindern. $\mathrm{Zu} 5 \mathrm{ml}$ Urinprobe in einem Reagenzglas gaben wir $5 \mathrm{~m} l$ Reagenz. Nach $10 \mathrm{Min}$. schüttelten wir mit $10 \mathrm{ml}$ Äthylacetat aus, pipettierten von der organischen Schicht $5 \mathrm{~m} l$ ab und schüttelten dièse in einem Scheidetrịchter mit $5 \mathrm{ml} 0,2$ proz. $\mathrm{NaHCO}_{3}$ aus. Die wäßr. Phase verdünnten wir 10 fach und photometrierten gegen 0,2 proz. $\mathrm{NaHCO}_{3}$ bei $380 \mathrm{~nm}$. Dazu bestimmten wir den Kreatiningehalt der Urinprobe und errechneten die Extinktion pro $\mathrm{mg} / 100 \mathrm{~m} /$ Kreatinin.

\section{Untersucbte Personengruppen}

Normalpersonen: Wir untersuchten drei Gruppen:

1. 5 Kinder bis etwa 1 Monat alt (Auftragsmenge entsprach $0,1 \mathrm{mg}$ Kreatinin)

2. 4 Kinder bis etwa 1 Jahr alt (Auftragsmenge entsprach 0,25 mg Kreatinin)

3. 4 Erwachsene zwischen 23 und 30 Jahren alt (Auftragsmenge entsprach $0,5 \mathrm{mg}$ Kreatinin).

Die Kinder der Gruppe 1 und 2 sind Patienten der Klinik, deren Krankheit wahrscheinlich keinen Einfluß auf die Ketosäuteausscheidung hatte, da sie sich im Zustand guter Kompensation befanden (blasse Asphyxic, ABO-Erythroblastose, Atemnotsyndrom einige Tage nach der Geburt, kongenitales Vitium, Kardiainsuffizienz, Dystrophie, $2 \times$ Pyelonephritis). Die erwachsenen Personen waren gesund.

Phenylketonurie: Wir untersuchten sieben Patienten mit unbehandelter oder ungenïgend behandelter Phenylketonurie (Phe-

nylalanin im Blut $20 \mathrm{mg} / 100 \mathrm{~m} / \mathrm{im}$ mikrobiologischen Hemmtest nach Gutrrie), 3 davon auch unter phenylalaninarmer Diät (Phenylalanin im Blut. $8 \mathrm{mg} / 100 \mathrm{~m} /$ ). Ein Patient wurde im Versuch mit $0,1 \mathrm{~g}$ Phenylalanin pro $\mathrm{kg} \mathrm{KG}$ belastet.

Cystinose: Wir untersuchten 6 Patienten:

1. K. F. 2 Jahre, behandelt

2. N. A. $21 / 2$ Jahre, behandelt

3. F. U. 1 Jahr, behandelt

4. Th. D. 4 Jahre, behandelt und unbehandelt

5. R. M. 9 Jahre, unbehandelt

6. O. U. 47 Jahre, unbehandelt.

Patient 6 ist einer der bei Erwachsenen sehr seltenen Fälle mit nachgewiesenen Cystinkristallen in Cornea und Conjunktiva. Die Behandlung der anderen Patienten bestand in einer Methioninund Cystin-armen Diät.

\section{Ergebnisse}

Wir testeten zahlreiche Fließmittelkombinationen auf Cellulose- und Kieselgelplatten. Bei der Kombination auf Kieselgelplatten, wie sie oben angegeben ist, erhielten wir die besten Ergebnisse. Trotz der unvermeidlichen Doppelflecke sind hier ein Großteil der Testsubstanzen getrennt (Abb. 1). Die Tabelle zeigt die $R_{\mathrm{F}}$-Werte der Testsubstanzen bei den verwendeten Fließmitteln.

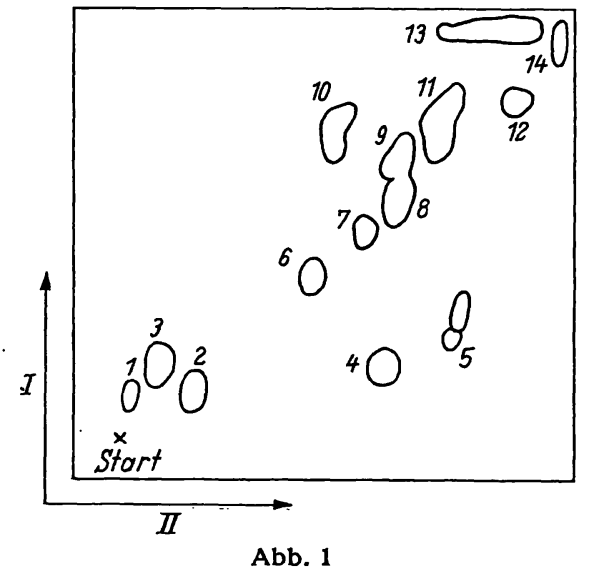

Verteilung der Testsubstanzen auf $10 \times 10 \mathrm{~cm}$ Kieselgel-Fertigplatten. Fließmittel wie im Text. Die Nummern entsprechen den Hydrazonen folgender Ketosäuren: 1. Oxalessigsäure -2. Glyoxylsäure I - 3 . 2-Oxo-glutarsäure - 4. Brenztraubensäure I - 5. Vereinte Doppelflecken $z$ u 8. 9. 10. 11. - 6. Glyoxylsäure II - 7. Brenztraubensäure II - 8. 2-Oxo-buttersäure - 9. 2-Oxo-4-methylmercapto-buttersäure - 10. 4-Hydroxy-phenylbrenztraubensäure - 11. Phenylbrenztraubensäure, 3-Methyl-2-oxo-pentansäure und 4-Methyl-2-oxo-pentansäure - 12. Laevulinsäure - 13. Ascorbinsäure - 14. 2,4-Dinitrophenyl-hydrazin

Tab. 1

$R_{F}$-Werte von 13 Hydrazonen bei eindimensionaler Chromatographie mit drei Fließmittelkombinationen auf Kieselgel-Fertigplatten (Fa. Merck, Charge Nr. 8488128 ).

Fließmittel A: Aceton/Isoamylalkohol/W'asser; 50:50:1 (v/v)

B: Isoamylalkohol/0,25N $\mathrm{NH}_{4} \mathrm{OH} ; 20: 1(\mathrm{v} / \mathrm{v})$

$\mathrm{SA}=$ Neigung zu Schwanzbildung bei Fließmittel A

$\mathrm{SB}=$ Neigung zu Schwanzbildung bei Fließmittel $\mathrm{B}$

${ }_{*}=$ die so gekennzeichnete Komponente überwiegt stark

\begin{tabular}{llll}
\hline 2,4-Dinitro-phenylhydrazone der: & A & B & C
\end{tabular}

Brenztraubensäure

Glyoxylsäure

Oxalessigsäure

2-Oxo-glutarsäure

2-Oxo-buttersäure (SA)

2-Oxo-4-methylmercapto-butter-

säure

Ascorbinsäure (SB)

Laevulinsäure

Phenylbrenztraubensäure

4-Hydroxy-phenylbrenztrauben-

säure

4-Methyl-2-oxo-pentansäure

3-Methyl-2-oxo-pentansäure

2,4-Dinitro-phenylhydrazin

\begin{tabular}{|c|c|c|}
\hline $\begin{array}{ll}0,11 & 0,47 \\
0,09 & 0,35 \\
0,06 & \\
0,12 & \\
0,17 & 0,54 *\end{array}$ & $\begin{array}{lll}0,15 & 0,13 \\
0,11 & 0,29 \\
0,00 & \\
0,06 & \\
0,20 & 0,35\end{array}$ & $\begin{array}{l}0,190,23 \\
0,120,77 \\
0,00 \\
0,06 \\
0,25 * 0,44\end{array}$ \\
\hline $\begin{array}{l}0,59 \\
0,99 \\
0,80 \\
0,20 \quad 0,65 *\end{array}$ & $\begin{array}{ll}0,32 & \\
0,82 & 0,91 \\
0,12 & \\
0,22 & 0,43^{*}\end{array}$ & $\begin{array}{l}0,22 \\
0,88 \\
0,65 \\
0,29 * 0,51\end{array}$ \\
\hline $\begin{array}{ll}0,22 & 0,68 * \\
0,24 & 0,71 * \\
0,26 & 0,70 * \\
0,99 & \end{array}$ & $\begin{array}{ll}0,24 & 0,40 * \\
0,25 & 0,48 * \\
0,48 & \\
0,82 & 0,91\end{array}$ & $\begin{array}{l}0,05 * 0,12 \\
0,35 * 0,57 \\
0,35 * 0,57 \\
0,88\end{array}$ \\
\hline
\end{tabular}




\section{Normalpersonen}

Die Chromatogramme ergaben bei verschiedenen Auftragskonzentrationen (s. oben) etwa gleiche Fleckenintensität, d. h. die bis zu einem Monat alten Kinder hatten eine größere Ausscheidung als die älteren und diese wieder (allerdings weniger ausgeprägt) als die Erwachsenen. Abbildung 2 zeigt das Chromatogramm eines

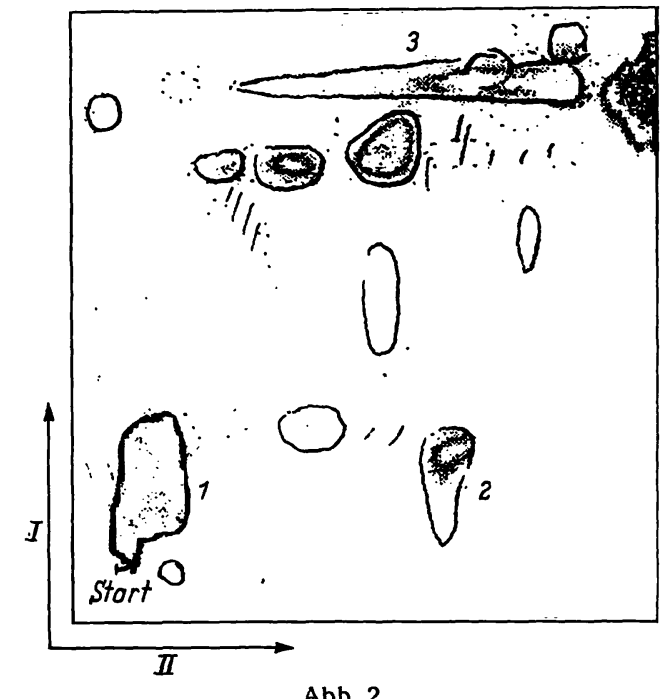

Trennung von Ketosäuren im Urin. 10 Tage alter Patient, ABO-Erythroblastose. Auftragsmenge $0,1 \mathrm{mg}$ Kreatinin entsprechend. Die Oxo-glutarsäure - 2. Brenztraubensäure - 3. Ascorbinsäure
O

10 Tage alten Säuglings (ABO-Erythroblastose) mit reichhaltigem Fleckenbild. Die Identifizierung gelang nur teilweise. Diese Untersuchungen dienten nur zum orientierenden Vergleich, wie sich aus der Zusammensetzung des untersuchten Personenkreises ergibt. Das genaue Muster, besonders der verschiedenen Altersstufen, muß noch bei einer großen Zahl Gesunder bestimmt werden.

\section{Pbenjlketonurie}

Die Chromatogramme der Extrakte aller unbehandelten Patienten zeigen ein seht gleichförmiges Ausscheidungsmuster bei hoher Intensität des Phenylbrenztraubensäurehydrazons, auch bei einer Auftragsmenge, die nur $0,1 \mathrm{mg}$ Kreatinin entsprach. Abbildung 3 zeigt das Chromatogramm eines 3 Jahre alten Patienten mit Phenylketonurie unter Phenylalaninbelastung. Es entspricht in Intensität und Verteilung der Flecke den anderen Chromatogrammen dieser Krankheit. Die Dreiergruppe zusammen mit dem starken Fleck rechts unten machen das typische Muster aus. Abbildung 4 zeigt das Chromatogramm desselben Patienten unter phenylalaninarmer Diät. Hier tritt der Fleck des Hydrazons der Brenztraubensäure deutlicher hervỏr. Eine Ausscheidung von Phenylbrenztraubensäure ist nicht mehr nachzuweisen. Das gleiche Ergebnis fanden wir bei allen drei vor und unter Behandlung untersuchten Patienten. Diese Chromatogramme sollen zeigen, daß sich mit der Methode Veränderungen der Ketosäureausscheidung zweifelsfrei nachweisen lassen.

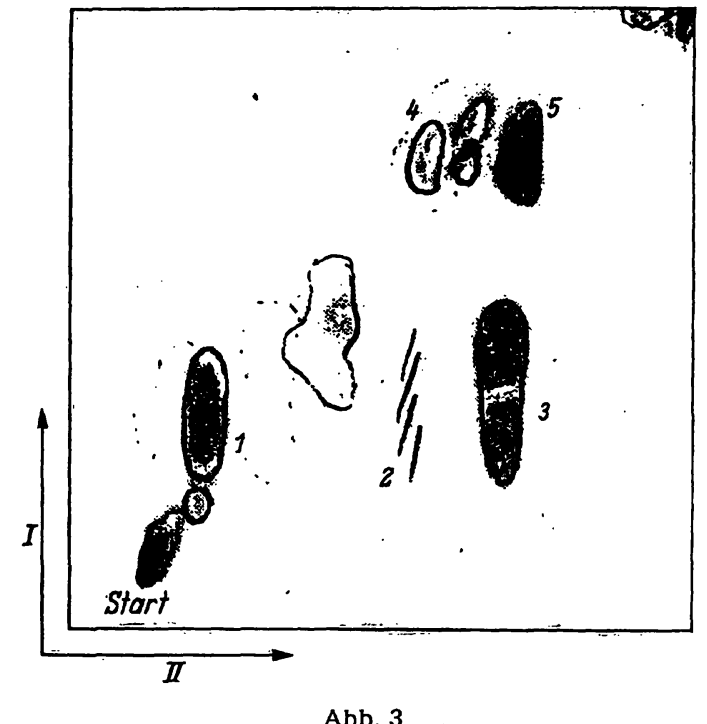

Trennung von Ketosäuren im Urin. Patient mit Phenylketonurie 3 Jahre alt. Auftragsmenge $0,25 \mathrm{mg}$ Kreatinin entsprechend. Die Nummern entsprechen den Hydrazonen folgender Ketosäuren: 1. 2 - 2. Brenztraubensäure - 3. Phenylbrenztraub säure I - 4. (chromatographisch identisch mit) 4-Hydroxy-phenylbrenztraubensäure -5 . Phenylbrenztraubensäure II

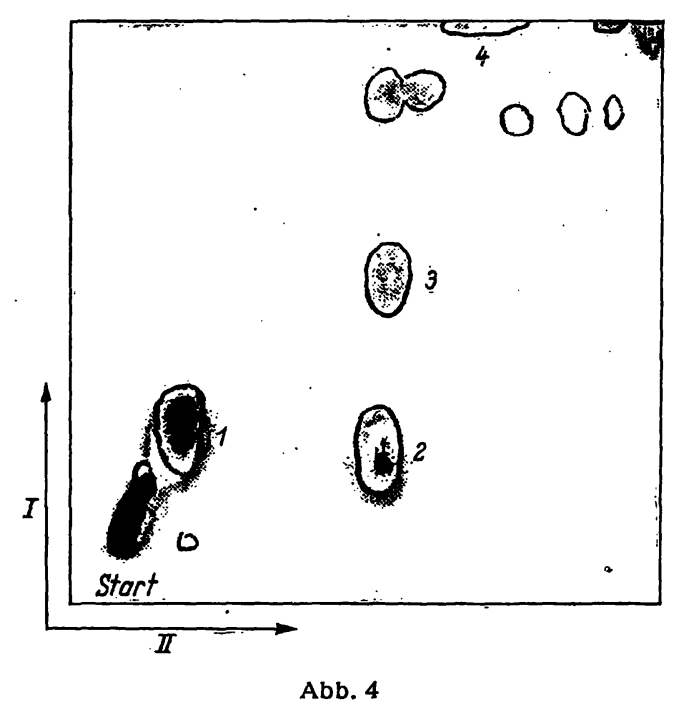

Trennung von Ketosäuren im Urin. Patient wie in Abb. 3 unter Behandlung. Auftragsmenge $0,125 \mathrm{mg}$ Kreatinin entsprechend. Die Nummern entsprechen den Hydrazonen folgender Ketosäuren: 1. 2-Oxo-glutarsäure -2 . Brenztraubensäure I - 3. Brenztraubensäure II -4 . Ascorbinsäure

\section{Cystinose}

SCHÄRER und ANTENER (8) fanden bei Cystinosepatienten eine erhöhte Ausscheidung nicht aminierter organischer Säuren, insbesondere Säuren des Citronensäurezyklus, darunter auch Ketosäuren. Besonders interessierten uns die 2-Oxo-buttersäure und 2-Oxo-4-methylmercapto-buttersäure (oxydativ desaminiertes Methionin), beide zum Methionin-Cystinstoffwechsel gehörend. Wir fanden bei den mit Methionin-Cystin-armer Diät behandelten Cystinosen ein'sehr gleichförmiges Muster (Abb. 5). Insgesamt ist die Ketosäureausscheidung stark erhöht, besonders aber die Ausscheidung von Brenztraubensäure und 2-Oxoglutarsäure. Die Gruppe von vier kleinen Flecken unterhalb des Fleckes des Ascớrbinsäurehydrazons ist konstant anzutreffen, konnte aber nicht zugeordnet werden. Sicher handelt es sich bei keinem der auf- 
BEHRINGWERKE AG

MARBURG-LAHN

St beching

\section{Plasmaprotein- Antisera Behringwerke

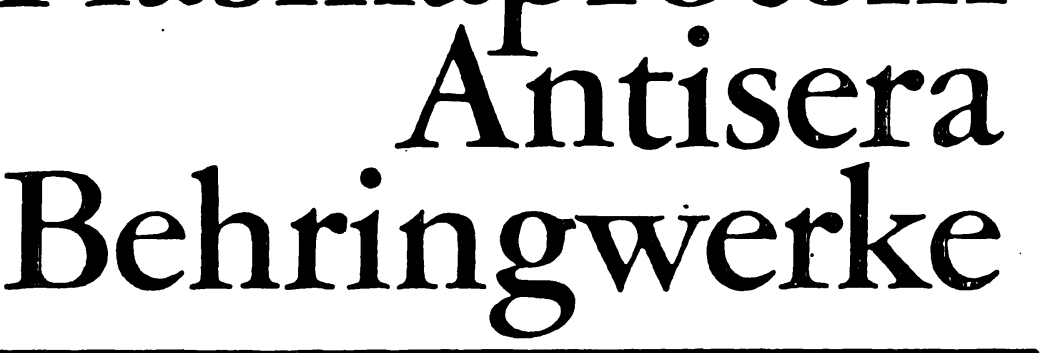

Qualitative und quantitative immunologische Bestimmung von Plasmaproteinen

Bluteiweiß-Differentialdiagnostik durch einfache Immunpräzipitations-Methoden

B64040

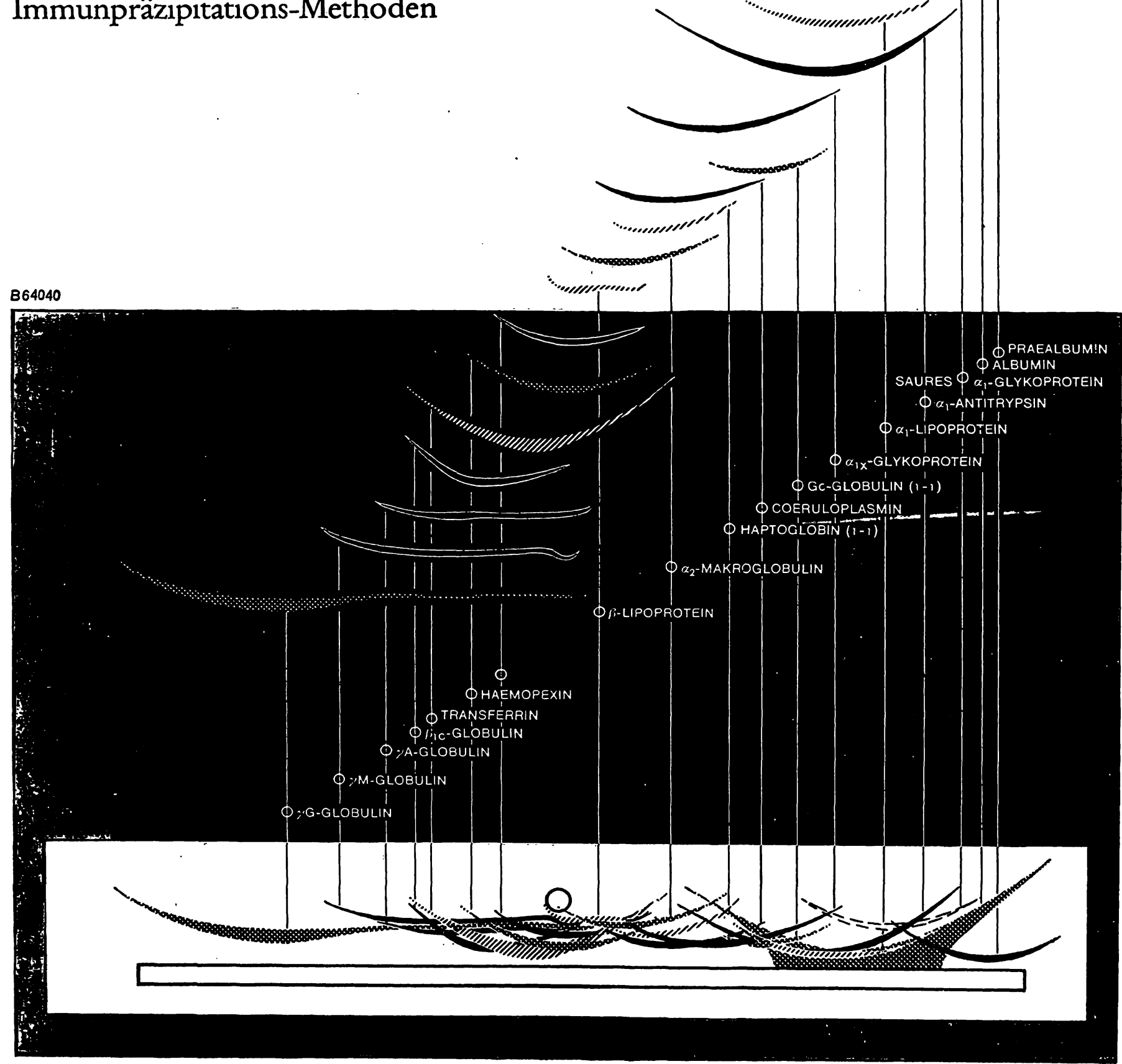


Aus Anlaß der hundertsten Wiederkehr des Jahres, , in dem zum ersten Mal Margarine hergestellt wurde, hat das Margarine-Institut für gesunde Ernährung, Hamburg, eine

\section{Studienförderung der}

\section{Ernährungswissenschaften}

ins Leben gerufen.

Die Studienförderung umfaßt zehn Stipendien, jedes gewährt auf zwei Jahre. Der monatliche Stipendienbetrag ist DM 400.

Durch diese Stipendien sollen begabte Doktoranden an deutschen Hochschulen gefördert werden, die sich auf eine Promotion über

chemische, biochemische, physiologische, medizinische Aspekte der menschlichen Ernährung, insbesondere über die Rolle der Fette in Ernährung und Stoffwechsel, vorbereiten. Die Bewerber bedürfen derEmpfehlung durch einen Hochschullehrer.

Über die Vergabe der Stipendien entscheidet ein Gutachterausschuß, der aus deutschen Hochschullehrern besteht. Ihm gehören gegenwärtig an:

$\begin{array}{ll}\text { Prof. Dr. W. Droese } & \text { Prof. Dr. G. Schettler } \\ \text { Prof. Dr. W. Heimann } & \text { Prof. Dr.Th.Wieland } \\ \text { Prof. Dr. J. Kühnau } & \text { Prof. Dr. V.Wolf } \\ \text { Prof. Dr. Dr. K. Lang } & \text { Prof. Dr. N.Zöllner }\end{array}$

Bewerbungen sind zu richten an den Beauftragten des Gutachterausschusses, Prof. Dr. Alfons Fricker, 7501 Grötzingen, Ringelberghohl 12 


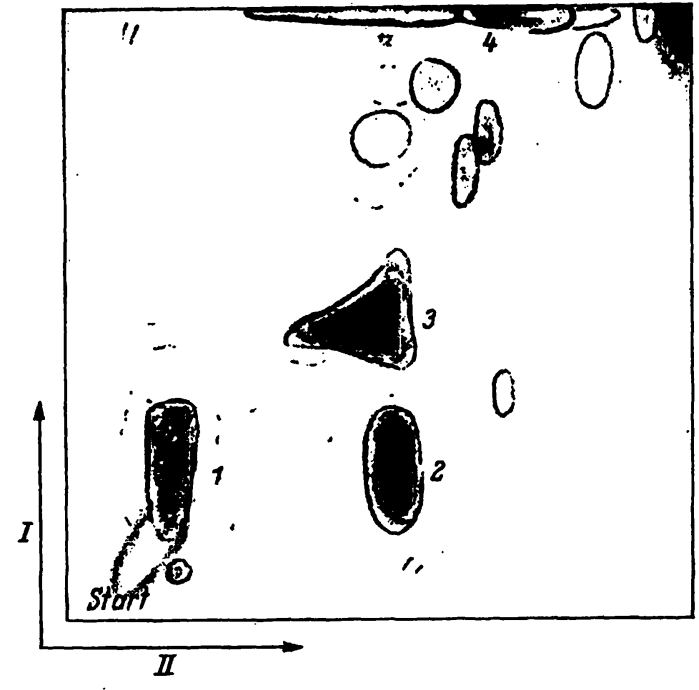

Abb. 5

Trennung von Ketosäuren im Urin. Patient mit Cystinose, $2^{i} / 2$ Jahre alt. Auftragsmenge 0,25 mg Kreatinin entsprechend. Die Nummern entsprechen den Hydrazonen folgender Ketosauren: 1. 2-Oxo-glutarAscorbinsäure tretenden Flecken um die Hydrazone der 2-Oxo-buttersäure und 2-Oxo-4-methylmercapto-buttersäure, wie die Zumischung authentischer Proben ergab. Die Darstellung von 2-Oxo-3-mercapto-propionsäure (oxydativ desaminiertes Cystein) bereitete bislang noch nicht überwundene Schwierigkeiten, so daß vergleichende Untersuchungen nicht möglich waren. Interessanterweise ist die Ketosäureausscheidung bei dem 47 jährigen Patienten (Nr. 6. s. oben) annähernd normal und stützt die Annahme eines andersartigen Speicherungsmechanismus (9). Im Suchtest lagen die Extinktionswerte bei den unbehandelten Cystinosen und dem einen unbehandelten Patienten (Nr. 5) zwischen $\mathrm{E}=0,02$ und 0,075 pro $\mathrm{mg} /$ $100 \mathrm{~m} l$ Kreatinin. Gesunde Kinder zeigten Werte von $\mathrm{E}=<0,01$ pro $\mathrm{mg} / 100 \mathrm{~m} /$ Kreatinin. Die unbehandelten Cystinosen ergaben ein uneinheitliches Bild, in keinem Fall aber eine größere Ketosäureausscheidung als unter der Behandlung. Es ist daher wahrscheinlich, $\mathrm{daß}$ die Methionin-Cystin-arme Diät die Ketosäureausscheidung nicht verringert.

\section{Literatur}

1. Cavalitini, D., N. Frontali und G. Toschi, Nature London 163, 568 (1949). - 2. CowARd, R. F. und P. SmIth, J. Chromatog. 33, 508 (1968). - 3. Berlet, H. H., Analytic. Biochem. 22, 525 (1968). - 4. Meister, A., J. biol. Chemistry 197, 308 (1952). 5. Hawary, M. F. S. und R. H. S. Thomson, Biochem. J. 53, 340 (1953). - 6. Meister, A., Biochemistry of the Amino Acids,
Vol. 1, S. 162, Second Edition, Academic Press New YorkLondon (1969). - 7. Bremer, H. J., W. Nützenadel und H. BICKEL, Mschr. Kinderheilk. 117, 32 (1969). - 8. SCHÄRER, K. und I. ANtener, Ann. paediatr. Basel, Suppl. 1 ad Vol. 203 (1964). - 9. Kraus, E., P. Lutz und H. BiCKeL, in Vorbereitung.

Dr. Dr. P. Lutz

69 Heidelberg

Hofmeisterweg $1-9$ 